\title{
Radiative Decays at LHCb
}

\section{Marius Ciprian Orlandea ${ }^{1}$}

National Institute for Physics and Nuclear Engineering (IFIN-HH)

E-mail: Marius.Orlandealcern.ch

We present a measurement of the ratio of the branching fractions of the radiative decays $\mathrm{B}^{0} \rightarrow \mathrm{K}^{* 0} \gamma$ and $\mathrm{B}_{\mathrm{s}}^{0} \rightarrow \phi \gamma$ using $1.0 \mathrm{fb}^{-1}$ of data taken with the LHCb detector. The value obtained is:

$$
\left.B\left(\mathrm{~B}^{0} \rightarrow \mathrm{K}^{* 0} \gamma\right) / B\left(\mathrm{~B}_{\mathrm{s}}^{0} \rightarrow \phi \gamma\right)=1.23 \pm 0.06 \text { (stat.) } \pm 0.04 \text { (syst. }\right) \pm 0.10\left(\mathrm{f}_{\mathrm{s}} / \mathrm{f}_{\mathrm{d}}\right) .
$$

Using the world average value $B\left(\mathrm{~B}^{0} \rightarrow \mathrm{K}^{* 0} \gamma\right)=(4.33 \pm 0.15) \times 10^{-5}$, the $\mathrm{B}_{\mathrm{s}}^{0} \rightarrow \phi \gamma$ branching fraction is determined to be $B\left(\mathrm{~B}^{0}{ }_{\mathrm{s}} \rightarrow \phi \gamma\right)=(3.5 \pm 0.4) \times 10^{-5}$. A measurement of the direct $\mathrm{CP}$ asymmetry of the decay $\mathrm{B}^{0} \rightarrow \mathrm{K}^{* 0} \gamma$ is also presented. Both measurements are the most precise to date and are in agreement with the previous experimental results and theoretical expectations.

14th International Conference on B-Physics at Hadron Machines April 8-12, 2013

Bologna, Italy

\footnotetext{
${ }^{1}$ Speaker

On behalf of the LHCb Collaboration
} 


\section{Introduction}

Radiative decays of the B-mesons provide a good test for the Standard Model (SM) as they can be described through one-loop penguin diagrams involving Flavour Changing Neutral Current (FCNC) processes. Measurement of the branching fractions of the radiative decays of the B mesons can give hints of the presence of new physics (NP) particles at the tree and loop levels. Gluon loop processes in $\mathrm{b} \rightarrow(\mathrm{s}, \mathrm{d})$ transitions give access to $\left|\mathrm{V}_{\mathrm{td}}\right|$ and $\left|\mathrm{V}_{\mathrm{ts}}\right|$, complement $\Delta \mathrm{m}_{\mathrm{d}, \mathrm{s}}$ measurements, and provide a direct constraint on the matrix CKM. Extra one-loop contributions from beyond the Standard Model (SM) process which affect the transition dynamics may be extracted from measurements $\mathrm{BR}, \mathrm{A}_{\mathrm{CP}}$, isospin asymmetries and the helicity structure of the photon.

This paper reports on the measurements of the branching fraction ratio $B\left(\mathrm{~B}^{0} \rightarrow \mathrm{K}^{* 0} \gamma\right) / B\left(\mathrm{~B}_{\mathrm{s}} \rightarrow \phi \gamma\right)$ using data corresponding to an integrated luminosity of $1.0 \mathrm{fb}^{-1}$ of $p p$ collision data collected by the LHCb experiment at a centre-of-mass energy of $7 \mathrm{TeV}$ in 2011. The measured ratio and the world average value of $B\left(\mathrm{~B}^{0} \rightarrow \mathrm{K}^{*} \gamma\right)$ are then used to determine $B\left(\mathrm{~B}^{0}{ }_{\mathrm{s}} \rightarrow \phi \gamma\right)$. A measurement of the direct $\mathrm{CP}$ asymmetry of the decay $\mathrm{B}^{0} \rightarrow \mathrm{K}^{* 0} \gamma$ is also presented. Further details can be found in [1].

\section{Detector and trigger strategy}

The LHCb detector, described in detail in Ref. [2], is a single-arm forward spectrometer covering the pseudorapidity range $2<\eta<5$, designed for the study of particles containing b or $\mathrm{c}$ quarks. The detector includes a high precision tracking system consisting of a silicon-strip vertex detector surrounding the $p p$ interaction region, a large-area silicon-strip detector located upstream of a dipole magnet with a bending power of about $4 \mathrm{Tm}$, and three stations of siliconstrip detectors and straw drift tubes located downstream. Charged hadrons are identified using two ring-imaging Cherenkov (RICH) detectors. Photon, electron and hadron candidates are identified by a calorimeter system consisting of scintillating-pad and preshower detectors, an electromagnetic calorimeter and a hadronic calorimeter. Muons are identified by a system composed of alternating layers of iron and multiwire proportional chambers.

The trigger consists of a hardware stage, based on information from the calorimeter and muon systems, followed by two software stages which apply a full event reconstruction. Decay candidates are required to have triggered on the signal photon and the daughters of the vector meson. At the hardware stage, the decay candidates must have been triggered by an electromagnetic candidate with transverse energy $\left(\mathrm{E}_{\mathrm{T}}\right)>2.5 \mathrm{GeV}$. At the first software stage, events are selected when a charged track is reconstructed with (impact parameter) IP $\chi^{2}>16$. Furthermore, a charged track is required to have either (transverse momentum) $\mathrm{P}_{\mathrm{T}}>1.7 \mathrm{GeV} / \mathrm{c}$ for a photon with $\mathrm{E}_{\mathrm{T}}>2.5 \mathrm{GeV}$, or $\mathrm{P}_{\mathrm{T}}>1.2 \mathrm{GeV} / \mathrm{c}$ when the photon has $\mathrm{E}_{\mathrm{T}}>4.2 \mathrm{GeV}$. At the second software stage, a track passing the previous criteria must form a $\mathrm{K}^{* 0}$ or $\phi$ candidate when combined with an additional track, and the invariant mass of the combination of the $\mathrm{K}^{* 0}(\phi)$ candidate and the photon candidate that triggered the hardware stage is required to be within 1 $\mathrm{GeV} / \mathrm{c}^{2}$ of the world average $\mathrm{B}^{0}\left(\mathrm{~B}_{\mathrm{s}}^{0}\right)$ mass. 


\section{Background sources}

The partial reconstruction of charged $\mathrm{B} \rightarrow \mathrm{h}^{+} \mathrm{h}^{-} \gamma \mathrm{X}$ or $\mathrm{B} \rightarrow \mathrm{h}^{+} \mathrm{h}^{-} \pi^{0} \mathrm{X}$ decays gives a broad background contribution at lower candidate masses, with a high-mass tail that extends into the signal region. The partially reconstructed $\mathrm{B}^{+} \rightarrow \mathrm{K}^{*} \pi^{+} \gamma$ and $\mathrm{B}^{+} \rightarrow \phi \mathrm{K}^{+} \gamma$ radiative decays produce a peaking contribution in the low-mass sideband at around $5.0 \mathrm{GeV} / \mathrm{c}^{2}$ for $\mathrm{B}^{0} \rightarrow \mathrm{K}^{* 0} \gamma$ and around $4.5 \mathrm{GeV} / \mathrm{c}^{2}$ for $\mathrm{B}_{\mathrm{s}}^{0} \rightarrow \phi \gamma$. The corresponding contamination from all the above sources has been estimated to be $(3.3 \pm 1.1) \%$ and $(1.8 \pm 0.3) \%$ for the $\mathrm{B}^{0} \rightarrow \mathrm{K}^{* 0} \gamma$ and $\mathrm{B}_{\mathrm{s}}{ }_{\mathrm{s}} \rightarrow \phi \gamma$ decays, respectively.

Contamination from several exclusive background decays is studied using simulated data samples. The irreducible $\mathrm{B}_{\mathrm{s}}^{0} \rightarrow \mathrm{K}^{*} \gamma$ decays, the $\Lambda_{\mathrm{b}}^{0} \rightarrow \Lambda^{*}\left(\mathrm{pK}^{-}\right) \gamma$ decays, and the charmless $\mathrm{B}_{\mathrm{s}}^{0} \rightarrow \mathrm{h}^{+} \mathrm{h}^{-} \pi^{0}$ decays produce peaked contributions under the invariant mass peak of $\mathrm{B}^{0} \rightarrow \mathrm{K}^{* 0} \gamma$. Additional contributions from the partial reconstruction of multi-body charmed decays and $\mathrm{B} \rightarrow \mathrm{V} \pi^{0} \mathrm{X}$ have been added to simultaneous fits to the data samples in the same way. The shape of these contributions is again determined from the simulation.

\section{Measurement of the ratio of branching fractions}

The ratio of branching fractions is measured as:

$$
\frac{B\left(\mathrm{~B}^{0} \rightarrow \mathrm{K}^{* 0} \gamma\right)}{B\left(\mathrm{~B}_{\mathrm{s}}^{0} \rightarrow \phi \gamma\right)}=\frac{\mathrm{N}_{\text {sig }}^{\mathrm{B}^{0} \rightarrow \mathrm{K}^{* 0} \gamma}}{\mathrm{N}_{\text {sig }}^{\mathrm{B}_{\mathrm{s}}^{0} \rightarrow \phi \gamma}} \times \frac{B\left(\phi \rightarrow \mathrm{K}^{+} \mathrm{K}^{-}\right)}{B\left(\mathrm{~K}^{* 0} \rightarrow \mathrm{K}^{+} \pi^{-}\right)} \times \frac{\mathrm{f}_{\mathrm{s}}}{\mathrm{f}_{\mathrm{d}}} \times \frac{\varepsilon_{\mathrm{B}_{\mathrm{s}}^{0} \rightarrow \phi \gamma}}{\varepsilon_{\mathrm{B}^{0} \rightarrow \mathrm{K}^{* 0} \gamma}}
$$

where $\mathrm{N}_{\text {sig }}$ are the observed yields of signal candidates, $B\left(\phi \rightarrow \mathrm{K}^{+} \mathrm{K}^{-}\right) / B\left(\mathrm{~K}^{* 0} \rightarrow \mathrm{K}^{+} \pi^{-}\right)=0.735 \pm$ 0.008 [3] is the ratio of branching fractions of the corresponding vector meson decays, $\mathrm{f}_{\mathrm{s}} / \mathrm{f}_{\mathrm{d}}=$ $0.267_{-0.020}^{+0.021}[4]$ is the ratio of the $\mathrm{B}^{0}$ and $\mathrm{B}_{\mathrm{s}}^{0}$ hadronization fractions in pp collisions at $\sqrt{\mathrm{s}}=7$ $\mathrm{TeV}$, and $\varepsilon_{\mathrm{B}_{\mathrm{S}}^{0} \rightarrow \phi \gamma} / \varepsilon_{\mathrm{B}^{0} \rightarrow \mathrm{K}^{* 0} \gamma}$ is the ratio of total reconstruction and selection efficiencies of the two decays. $\mathrm{N}\left(\mathrm{B}_{\mathrm{s}}^{0} \rightarrow \phi \gamma\right) / \mathrm{N}\left(\mathrm{B}^{0} \rightarrow \mathrm{K}^{*} \gamma\right)$ was obtained from the fit Shown in Fig. 1.
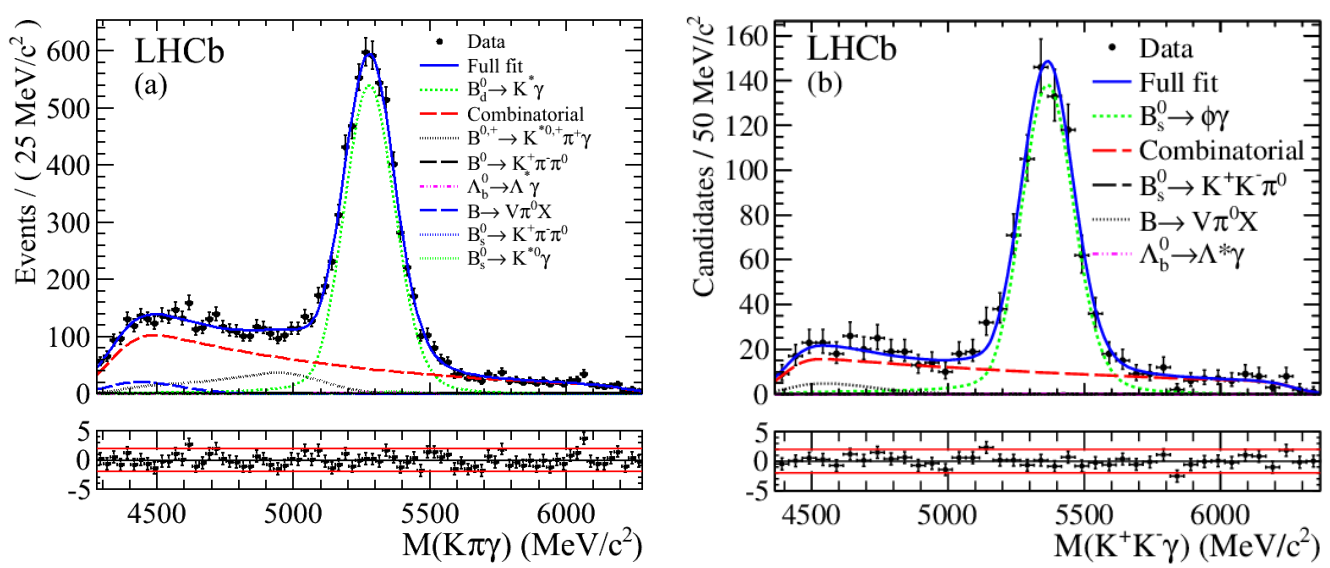

Fig.1: Distributions of the $\mathrm{B}^{0} \rightarrow \mathrm{K}^{* 0} \gamma$ (left) and $\mathrm{B}_{\mathrm{s}}^{0} \rightarrow \phi \gamma$ (right) candidates.

The efficiency ratio is:

$$
\frac{\varepsilon_{\mathrm{B}_{\mathrm{S}}^{0} \rightarrow \phi \gamma}}{\varepsilon_{\mathrm{B}^{0} \rightarrow \mathrm{K}^{* 0} \gamma}}=\mathrm{r}_{\text {reco\&sel }} \times \mathrm{r}_{\mathrm{PID}} \times \mathrm{r}_{\text {trigger }}
$$


where $r_{\text {reco\&sel }}, \quad r_{P I D}$ and $r_{\text {trigger }}$ are the efficiency ratios due to the reconstruction and selection, the particle identification (PID) and the trigger requirements, respectively.

\section{Measurement of the $C P$ asymmetry in $B^{0} \rightarrow K^{* 0} \gamma$ decays}

The physical $\mathrm{CP}$ asymmetry $\left(\mathrm{A}_{\mathrm{CP}}\right)$ is measurement as:

$$
A_{C P}\left(B^{0} \rightarrow K^{* 0} \gamma\right)=A_{R A W}\left(B^{0} \rightarrow K^{* 0} \gamma\right)-A_{D}(K \pi)-\kappa \times A_{P}\left(B^{0}\right)
$$

where the first term of the equation is the raw asymmetry in the final state, which must be corrected for detection and production effects. The next two terms, $A_{D}(K \pi)$ and $A_{P}\left(B^{0}\right)$, represent the detection asymmetry of the kaon and pion pair and $\mathrm{B}^{0}$ meson production asymmetry, respectively. The dilution factor $\kappa$ arises from the oscillations of neutral B mesons. Figure 2 shows the results of the fit. The fit includes product of corrections to the background model, magnet polarity, $\mathrm{B}^{0}$ meson production, and particle detection in final state.
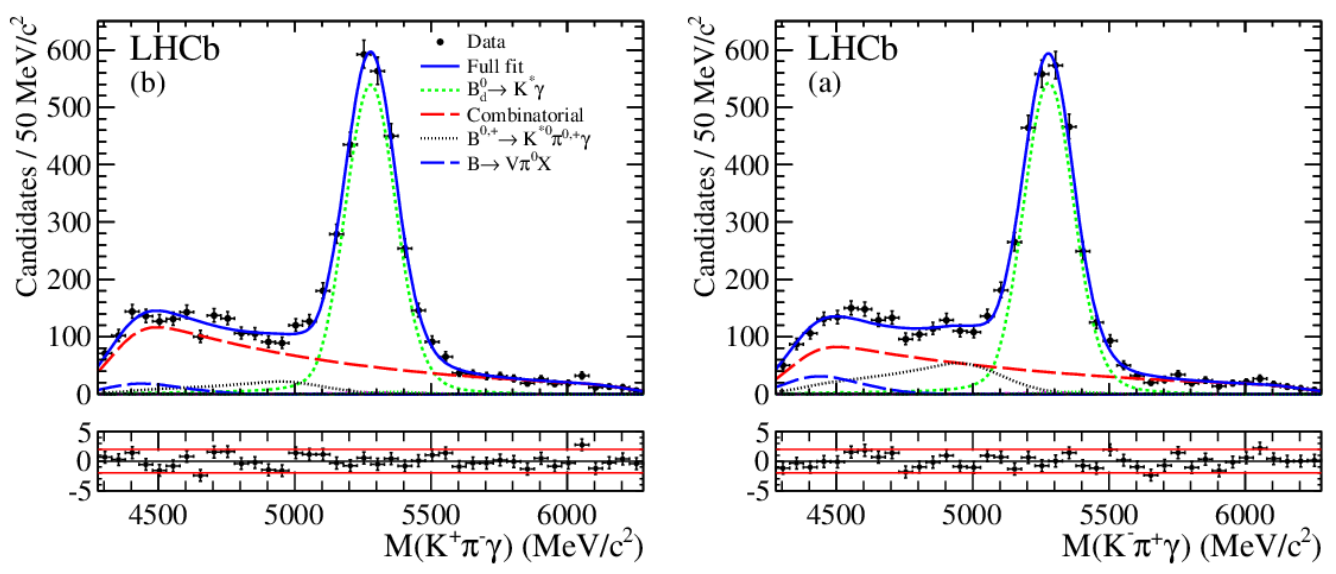

Fig.2: Distributions of the $\mathrm{B}^{0} \rightarrow \mathrm{K}^{* 0} \gamma$ (left) and $\overline{\mathrm{B}^{0}} \rightarrow \overline{\mathrm{K}^{* 0}} \gamma$ (right) decay candidates.

\section{Results and conclusions}

The ratio of branching fractions $\mathrm{B}^{0} \rightarrow \mathrm{K}^{* 0} \gamma$ and $\mathrm{B}_{\mathrm{s}}^{0} \rightarrow \phi \gamma$ has been measured to be:

$$
\left.\left.\frac{B\left(\mathrm{~B}^{0} \rightarrow \mathrm{K}^{* 0} \gamma\right)}{B\left(\mathrm{~B}_{\mathrm{s}}^{0} \rightarrow \phi \gamma\right)}=1.23 \pm 0.06 \text { (stat. }\right) \pm 0.04 \text { (syst. }\right) \pm 0.10\left(\frac{\mathrm{f}_{\mathrm{s}}}{\mathrm{f}_{\mathrm{d}}}\right)
$$

which is the most precise measurement to date and is in good agreement with the SM prediction of $1.0 \pm 0.2[5]$.

The branching fractions of $B\left(\mathrm{~B}_{\mathrm{s}}^{0} \rightarrow \phi \gamma\right)$ has been determined using the world average value of $B\left(\mathrm{~B}^{0} \rightarrow \mathrm{K}^{* 0} \gamma\right)=(4.33 \pm 0.15) \times 10^{-5}$ to be:

$$
B\left(\mathrm{~B}^{0}{ }_{\mathrm{s}} \rightarrow \phi \gamma\right)=(3.5 \pm 0.4) \times 10^{-5} .
$$

The direct $\mathrm{CP}$ asymmetry in $\mathrm{B}^{0} \rightarrow \mathrm{K}^{* 0} \gamma$ decays has also been measured with the same data sample and found to be: 


$$
\left.\mathrm{A}_{\mathrm{CP}}\left(\mathrm{B}^{0} \rightarrow \mathrm{K}^{* 0} \gamma\right)=(0.8 \pm 1.7 \text { (stat.) } \pm 0.9 \text { (syst. })\right) \%
$$

in agreement with the SM expectation of $(-0.61 \pm 0.43) \%$ [6].

\section{Acknowledgments}

We express our gratitude to our colleagues in the CERN accelerator departments for the excellent performance of the LHC. We thank the technical and administrative staff at CERN and at the LHCb institutes, and acknowledge support from the National Agencies: CAPES, CNPq, FAPERJ and FINEP (Brazil); CERN; NSFC (China); CNRS/IN2P3 (France); BMBF, DFG, HGF and MPG (Germany); SFI (Ireland); INFN (Italy); FOM and NWO (The Netherlands); SCSR (Poland); ANCS (Romania); MinES of Russia and Rosatom (Russia); MICINN, XuntaGal and GENCAT (Spain); SNSF and SER (Switzerland); NAS Ukraine (Ukraine); STFC (United Kingdom); NSF (USA). We also acknowledge the support received from the ERC under FP7 and the Region Auvergne.

\section{References}

[1] LHCb Collaboration R Aaij et al., Measurement of the ratio of branching fractions $B\left(B^{0} \rightarrow K^{x^{0}} \gamma\right)$ / $B\left(B_{s}^{0} \rightarrow \phi \gamma\right)$ and the direct $C P$ asymmetry in $B^{0} \rightarrow K^{x^{0}} \gamma$, Nuclear Physics B Volume 867 (2013) 1-18

[2] LHCb Collaboration, A.A. Alves Jr., et al., The LHCb detector at the LHC, JINST 3 (2008) S08005.

[3] Particle Data Group, J. Beringer, et al., Review of particle physics, Phys. Rev. D 86 (2012) 010001

[4] LHCb Collaboration, R. Aaij, et al., Measurement of b hadron production fractions in $7 \mathrm{TeV} p p$ collisions, Phys.Rev. D 85 (2012) 032008, [arXiv:1111.2357] .

[5] A. Ali, B.D. Pecjak, C. Greub, Towards $B \rightarrow V \gamma$ decays at NNLO in SCET, Eur. Phys. J. C 55 (2008) 577, [arXiv:0709.4422].

[6] M. Matsumori, A.I. Sanda, Y.Y. Keum, $C P$ asymmetry, branching ratios and isospin breaking effects of $B^{0} \rightarrow K^{* 0} \gamma$ with perturbative QCD approach, Phys. Rev. D 72 (2005) 014013, [arXiv:hep-ph/0406055] 